This item was submitted to Loughborough's Research Repository by the author.

Items in Figshare are protected by copyright, with all rights reserved, unless otherwise indicated.

\title{
Pyrene-based aggregation-induced emission luminogens (AIEgen): structure correlated with particle size distribution and mechanochromism
}

PLEASE CITE THE PUBLISHED VERSION

https://doi.org/10.1039/c9tc01665a

\section{PUBLISHER}

(C) Royal Society of Chemistry (RSC)

\section{VERSION}

AM (Accepted Manuscript)

\section{PUBLISHER STATEMENT}

This paper was accepted for publication in the journal Journal of Materials Chemistry $\mathrm{C}$ and the definitive published version is available at https://doi.org/10.1039/c9tc01665a.

\section{LICENCE}

CC BY-NC-ND 4.0

\section{REPOSITORY RECORD}

Feng, Xing, Jun Zhang, Zhen Hu, Qingsong Wang, Md. Monarul Islam, Jen-Shyang Ni, Mark Elsegood, Jacky W.Y. Lam, Erjun Zhou, and Ben Zhong Tang. 2019. "Pyrene-based Aggregation-induced Emission Luminogens (aiegen): Structure Correlated with Particle Size Distribution and Mechanochromism". Loughborough University. https://hdl.handle.net/2134/38108. 
eceived 00th January 20xx, Accepted 00th January 20xx DOI: $10.1039 / x 0 x \times 00000 x$

\title{
Pyrene-Based Aggregation-Induced Emission Luminogens (AIEgen): Structure Correlated with Particle Size Distribution and Mechanochromism
}

\author{
Xing Feng, ${ }^{*}{ }^{a b} \ddagger$ Jun Zhang, ${ }^{b} \ddagger$ Zhen Hu, ${ }^{a}$ Qingsong Wang, ${ }^{a}$ Md. Monarul Islam, ${ }^{a}$ Jen-Shyang Ni, ${ }^{c}$ Mark

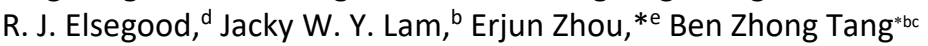

\begin{abstract}
This article describes a new schematic strategy for the preparation of pyrene-based aggregation-induced emission (AIE) luminogens, which show a potential application in smart materials. Utilising a restricted intramolecular rotation (RIR) mechanism, three non-typical pyrene-based luminogens 2-4 were synthesized and their AIE characteristics and mechanochromism (MC) were analysed in relation to their particle size distribution. The morphology of the synthesized 2 4 luminogens was investigated by scanning electron microscopy (SEM), transmission electron microscopy (TEM) and dynamic light scattering (DLS). The morphology of AlEgens $\mathbf{2}$ were changed from a gel-like species to small irregular patches and then to uniform, round-shaped nano-particle species through a crystal phase transition from the ordered packing structure to amorphous as the water fraction $\left(f_{w}\right)$ was increased. The different size distribution and morphological changes of nano-particle species plays a signficant role in enhancing the emission intensity in the aggregate state. X-ray single crystal diffraction shows that both compounds $\mathbf{2}$ and $\mathbf{4}$ have a twisted conformation with large dihedral angle between the pyrene core and the peripheral aromatic substituent group. The morphologies were further characterized by powder X-ray diffraction (PXRD) before and after grinding, which showed that the external force destroys the weak intermolecular interactions and leads to a change to a more planar molecular conformation. The minor change in packing pattern contributes to influence the emissive intensity as well as emission colour. As a result, we observed more molecules aggregating, more nanoparticle homogeneity and more shine.
\end{abstract}

\section{Introduction}

Pyrene and its derivatives are significant members of the family of polycyclic aromatic hydrocarbons (PAHs) which consist of four fused aromatic rings with strong $\pi$-electron delocalization characteristics. ${ }^{[1-2]}$ The basic properties of pyrenes with high charge-carrier mobility and excellent fluorescence, make them potential candidates for application in organic semiconductor materials, chemosensors, biolabeling, etc. However, the planar structure of pyrenes

a. Guangdong Provincial Key Laboratory of Functional Soft Condensed Matter, School of Material and Energy, Guangdong University of Technology, Guangzhou 510006, P. R. China. E-mail: hyxhn@sina.com.

b. Department of Chemistry and Hong Kong Branch of Chinese National Engineering Research Center for Tissue Restoration and Reconstruction, The Hong Kong University of Science \& Technology, Clear Water Bay, Kowloon, Hong Kong, China. E-mail: tangbenz@ust.hk.

c. HKUST-Shenzhen Research Institute, No. 9 Yuexing 1st RD, South Area, Hi-tech Park, Shenzhen 518057, China

d. Chemistry Department, Loughborough University, Loughborough LE11 3TU, UK.

e. CAS Key Laboratory of Nanosystem and Hierarchical Fabrication, CAS Center for

Excellence in Nanoscience, National Center for Nanoscience and Technology, Beijing 100190, China.E-mail: zhouej@nanoctr.cn

+ Electronic Supplementary Information (ESI) available: Materials and Methods; the ${ }^{1} \mathrm{H} /{ }^{13} \mathrm{C}$ NMR spectra of the new compounds; absorption and fluorescence spectra, DFT calculation data and EL properties (PDF) as Supporting Information are included. See DOI: 10.1039/x0xx00000x

$\ddagger \mathrm{X}$. Feng and J. Zhang contributed equally to this work. results in the aggregation-caused quenching (ACQ) phenomenon. ${ }^{[3]} \mathrm{A}$ high intensity blue emission is observed in dilute solution, but in contrast, the fluorescence is quenched in the aggregated state when the concentration is increased. This ACQ phenomenon has limited any high-technological application in organic electronics, i.e. organic light-emitting diodes (OLED).[1-2] Discovery of how to overcome the ACQ effect and further facilitate the development of pyrene-based materials for potential application in organic semiconductor and other fields of material chemistry, is a fundamental and demandable research topic in pyrene chemistry.

In 2001, Tang observed an abnormal optical phenomenon of aggregation induced emission (AIE) that the chromophore, such as hexaphenylsilole (HPS), ${ }^{[3]}$ or tetraphenylethylene (TPE), shows stronger emission in the solid (aggregation) state rather than in solution, and the mechanism was explained by restricted intramolecular rotation $(R I R),{ }^{[4]}$ in the solid state. The unrestricted intramolecular rotation of the molecule can open up the nonradiative pathway and result in non-emissive phenomenon; on the contrary, the nonradiative pathway would be blocked and opens up the radiative channel in the aggregated state. It is reported that the ACQ effect of traditional pyrene luminogens would be solved by introducing twist via bulky units, according to a RIR mechanism ${ }^{[5]}$. Previously, pyrene-based luminogens with three dimensional tetraphenylethylene (TPE) units ${ }^{[6]}$ or pyrene-based like- 
TPE luminogens, ${ }^{[7]}$ as in artificial organic dyes, were shown to exhibit clear AIE characteristics. These typical AIEgens showed negligible emission in solution but show high quality luminescence with high quantum yield in the solid state or in aggregation. For example, the 1,3,6,8-tetrakis[4-(1,2,2-triphenylvinyl)phenyl] pyrene (TTPEPY) exhibited bright fluorescence emission in the solid state with a high quantum yield of 0.7.[6] On the other hand, it is found that the twisted molecules (such as TPE-based luminogens) with AIE features prefer to exhibit polymorph-dependent mechanoluminescence (ML) properties. ${ }^{[8]}$ So, is it possible to design a planar structure with $\mathrm{ML} / \mathrm{MC}$ properties, such as pyrene-based AlEgens? Li et al. realised that two planar pyrene-based precursors (4-bromopyrene and 4-Bpinpyrene) would generate non-typical organic mechanoluminescence/mechanochromism (ML/MC) luminogens due to their different molecular packing, but this is a rare example in photochemistry. ${ }^{[9]}$

Until now, the design and synthesis of new pyrene-based AIEgen systems remains a challenge, and their $\mathrm{ML} / \mathrm{MC}$ properties have rarely been investigated. Kanvah et al. reported a series of pyrene-based cyanostyrene derivatives which show clear AIE characteristics with a dimer emission in aggregates; ${ }^{[10]}$ and $\mathrm{Chi}$ et al. summarized a series of $\mathrm{ML} / \mathrm{MC}$ of cyanoethylene derivatives with AIE properties. ${ }^{[11]}$ To understand in-depth the correlation of structure (particle size) with optical properties such as PL and MC, three new pyrenebased derivatives (2-4) were prepared and are presented in Scheme 1 . The detailed emission properties were examined by photoluminescence (PL) spectra and the morphology of aggregates was measured by powder X-ray diffraction (XRD) analyses, scanning electron microscopy (SEM), transmission electron microscopy (TEM) and dynamic light scattering (DLS). We observed that the morphology of the synthesized 2-4 luminogens plays a significant role affecting the photophysical properties, such as the photoluminescence behavior, PL quantum yield ( $Q Y$ ) and fluorescence lifetimes. In addition, the pyrene-based cyanostyrene $\mathbf{2}$ exhibits MC properties with tunable emission color upon mechanical stimulation.

\section{Results and discussion}

\section{Synthetic procedures}

The target compounds 2-3 were synthesized by Knoevenagel reaction between 2,7-di-tert-butylpyrene 4carbaldehyde (1) and the corresponding benzyl cyanide in respectable yield and are shown in scheme 1 . The direct condensation of $\mathbf{1}$ with 2,6-diisopropylaniline afforded the Schiff-base 4 in $65 \%$ yield. We designed the target compounds containing tert-butyl groups which play an important role in suppressing the $\pi-\pi$ stacking in solid state. Additionally the isopropyl groups were introduced into the 1,3-positions of the aromatic ring as bulky blocking groups for the further suppression of $\pi-\pi$ interactions of the pyrene unit in compound 4. ${ }^{[12]}$ The products $\mathbf{2}$ and $\mathbf{4}$ are soluble in common organic solvents such as dichloromethane $\left(\mathrm{CH}_{2} \mathrm{Cl}_{2}\right)$, tetrahydrofuran (THF), N,N-di-methylformamide (DMF) and 1,4-dioxane, but $\mathbf{3}$ has lower solubility, which may be due to the extended $\pi$-conjugation. The structures of all products were fully characterized by ${ }^{1} \mathrm{H} /{ }^{13} \mathrm{C} \quad \mathrm{NMR}$ and HRMS spectroscopy and, in the cases of $\mathbf{2}$ and $\mathbf{4}$, also by single crystal X-ray diffraction.

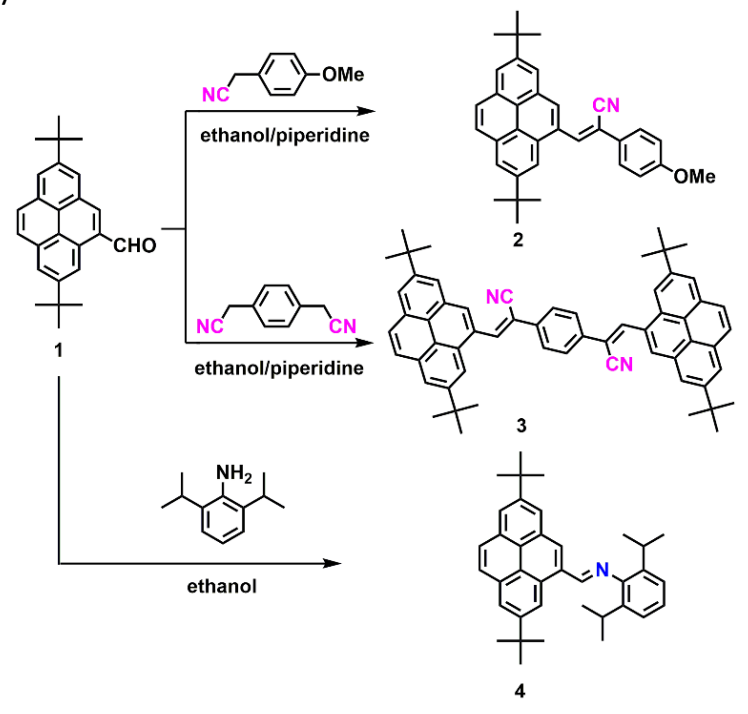

Scheme 1. Synthetic route to pyrene-based derivatives 2-4.

\section{Photophysical properties}

The absorption spectra of pyrene derivatives 2-4 were recorded in dilute THF solution and are displayed in Figure 1. Compounds $\mathbf{2}$ and $\mathbf{3}$ show identical absorption bands, with a strong absorption band at around $250-350 \mathrm{~nm}$ with a shoulder peak at around $350-450 \mathrm{~nm}$. The maximum absorption band of $\mathbf{3}$ shows a slight red-shift compared with $\mathbf{2}$, due to the extended $\pi$-conjugation in the molecular skeleton. It is noted that the Schiff-base 4 exhibits quite different absorption profiles in comparison with compounds $\mathbf{2}$ and $\mathbf{3}$. Compound $\mathbf{4}$ has two absorption peaks centred at $297 \mathrm{~nm}$ and $358 \mathrm{~nm}$. This difference may be due to the presence of the $\mathrm{CH}=\mathrm{N}$ group at the 4-position of the pyrene ring leading to delocalization of the $\pi$-electron density of the pyrene skeleton. The maximum absorption coefficients $(\varepsilon)$ were $24700 \mathrm{M}^{-1} \mathrm{~cm}^{-1}$ for 2, 79600 $\mathrm{M}^{-1} \mathrm{~cm}^{-1}$ for 3 and $35900 \mathrm{M}^{-1} \mathrm{~cm}^{-1}$ for 4 , respectively. Interestingly, the molecular framework of $\mathbf{3}$, containing two pyrenes, shows more than double the absorption coefficient compared with $\mathbf{2}$, which has only one pyrene unit.[13]

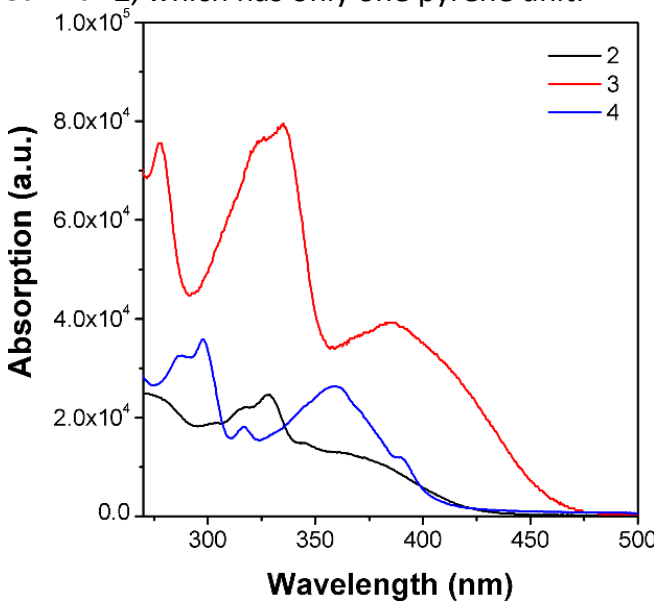

Figure 1. UV-vis spectra of 2-4 in THF solvent. 
Table 1. The photophysical properties of compounds 2-4.

\begin{tabular}{|c|c|c|c|c|c|c|c|c|}
\hline $\begin{array}{c}\text { Comd } \\
.\end{array}$ & $\begin{array}{c}\lambda_{\max a b s}{ }^{a} \\
n m\end{array}$ & $\begin{array}{c}\lambda_{\operatorname{maxPL}} \\
(\mathrm{nm})\end{array}$ & $\Phi_{f}$ & $\iota(n s)$ & $\begin{array}{l}\text { LUMO } \\
(\mathrm{eV})\end{array}$ & $\begin{array}{c}\text { HOMO } \\
(\mathrm{eV})\end{array}$ & $\begin{array}{c}\mathrm{Eg} \\
(\mathrm{eV})\end{array}$ & Tgf/Tdg \\
\hline 2 & 327 & $\begin{array}{c}461 \text { (in THF) } \\
525 \text { (99\%) } \\
507 \text { (crystal) } \\
500 \text { (grind) }\end{array}$ & $\begin{array}{c}0.03(0 \%) \\
0.20(99 \%) \\
22.1 \text { (crystal) } \\
23.3 \text { (grind) } \\
28.3 \text { (heavy grind) }\end{array}$ & $\begin{array}{c}5.8(99 \%) \\
2.45 \text { (crystal) } \\
3.07 \text { (grind) }\end{array}$ & $-2.04^{b} /-2.91^{d}$ & $-5.37^{b} /-5.72^{c}$ & $3.33 / 2.81^{\mathrm{e}}$ & $223 / 338$ \\
\hline 3 & 335 & $\begin{array}{c}505 \text { (in THF) } \\
540 \text { (99\%) } \\
538 \text { (powder) } \\
538 \text { (grind) }\end{array}$ & $\begin{array}{c}0.1 \text { (before) } \\
0.14 \text { (grinding) }\end{array}$ & $\begin{array}{l}1.57 \text { (powder) } \\
2.44 \text { (grinding) }\end{array}$ & $-2.50 /-2.71$ & $-5.46 /-5.70$ & $2.96 / 2.99$ & $--/ 439$ \\
\hline 4 & 297,358 & $\begin{array}{c}429 \text { (in THF) } \\
487(70 \%) \\
470(99 \%)\end{array}$ & $\begin{array}{c}0.01(0 \%) \\
0.06(70 \%) \\
0.01(99 \%)\end{array}$ & $\begin{array}{l}1.86(0 \%) \\
11.10(70) \\
4.16(99 \%)\end{array}$ & $-1.94 /-3.07$ & $-5.49 /-5.71$ & $3.55 / 2.64$ & $189 / 287$ \\
\hline $\begin{array}{l}{ }^{a} \text { Maxin } \\
\text { empiric } \\
\text { obtain } \\
\text { thermc }\end{array}$ & $\begin{array}{l}\text { m absorpt } \\
\text { I formula } 1 \\
\text { from diffe } \\
\text { ravimetric }\end{array}$ & $\begin{array}{l}\text { wavelength } n \\
10=\_(\text {Eox }+4.8 \\
\text { tial scanning } \\
\text { lysis (TGA). }\end{array}$ & $\begin{array}{l}\text { asured in } \mathrm{THF} \text { at roo } \\
\mathrm{LUMO}=\mathrm{HOMO}+\mathrm{Eg} \\
\text { orimetry (DSC) mea }\end{array}$ & $\begin{array}{l}n \text { temperature. }{ }^{b} \\
{ }^{e} \text { Eg estimated frc } \\
\text { urement. }{ }^{9} \text { Decon }\end{array}$ & $\begin{array}{l}\text { FT (B3LYP/6-31 } \\
\mathrm{m} \text { UV/Vis abso } \\
\text { position tempe }\end{array}$ & $\begin{array}{l}\text { calculations. } \\
\text { on spectra. }{ }^{f} \mathrm{~N} \\
\text { ure }\left(T_{\mathrm{d}}\right) \text { obtain }\end{array}$ & $\begin{array}{l}\text { Calculated } f \\
\text { Iting tempe } \\
\text { from }\end{array}$ & $\begin{array}{l}\mathrm{n} \text { the } \\
\text { ure }\left(T_{\mathrm{m}}\right)\end{array}$ \\
\hline
\end{tabular}

\section{AIE properties}

Due to the flat structure of pyrene, which tends to suffer ACQ problems in the solid state, manifested as a quench in fluorescence processes, many research groups have reported different approaches to achieve AlEgens of pyrene molecules such as i) introducing the tri-TPE/TPE into pyrene, ${ }^{[5 b, 14]}$ ii) construction of the pyrene-based ESIPT system ${ }^{[5 b, 15]}$ and iii) combing highly twisted moieties into the pyrene core. ${ }^{[16]}$ Based on our knowledge, the phenylacetonitrile moiety is widely employed for preparation of AIE-active materials. ${ }^{[11]}$

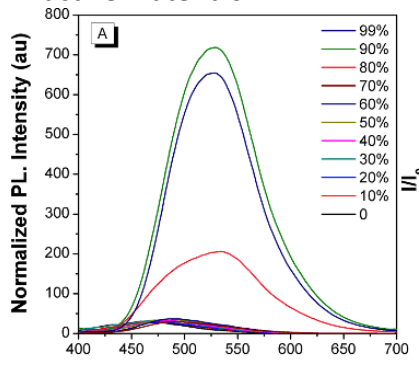

Wavelength $(\mathrm{nm})$

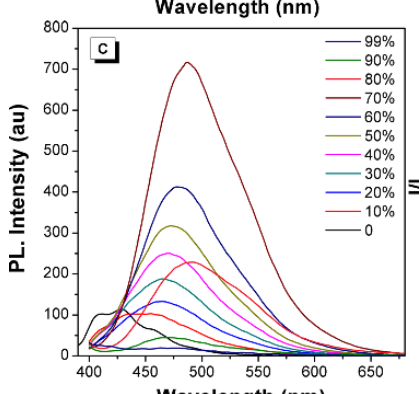

Wavelength $(\mathrm{nm})$
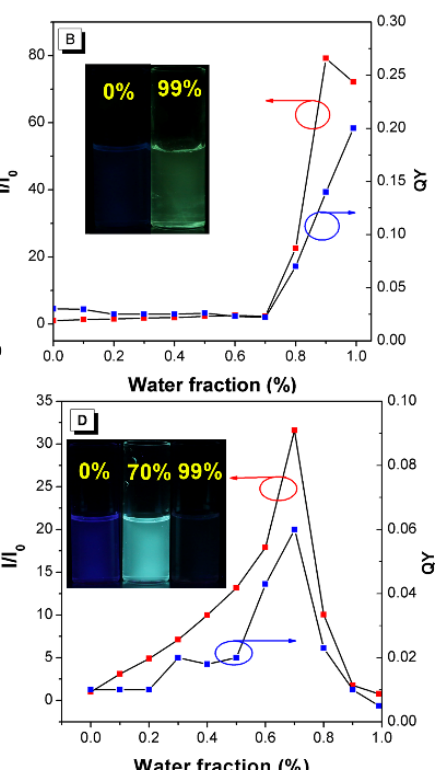

Figure 2. (A), (C) PL spectra of $\mathbf{2}$ and $\mathbf{3}$ in THF/water mixtures with different water fractions $\left(f_{\mathrm{w}}\right)$; (B), (D) left: Plot of relative $\mathrm{PL}$ intensity $\left(I / I_{0}\right)$ and right: Plot of relative QY versus the composition of THF/water mixture of $\mathbf{2}$, where $I_{0}$ is the PL intensity in pure THF solution. Insert (B) and (D): Fluorescent photographs of $\mathbf{2}$ and $\mathbf{3}$ in THF/water mixtures from $f_{\mathrm{w}}=0 \%(70 \%)$ and $99 \%$ taken under UV illumination ( $\lambda \mathrm{ex}=365 \mathrm{~nm}$ ).
To test the AIE properties, the emission behavior of $\mathbf{2}$ and $\mathbf{4}$ $(10 \mu \mathrm{M})$ in dilute THF and THF/water mixtures were recorded. The concentration of 3 is $2.5 \mu \mathrm{M}$ due to the poor solubility. The compound $\mathbf{2}$ emitted weak blue fluorescence $(460 \mathrm{~nm})$ in dilute THF solution, and shows bright emission as water is gradually added to the THF solution. When the fraction of water $\left(f_{w}\right)$ increases to $70 \%$, the fluorescence intensity becomes stronger and this was accompanied by a slightly red-shifted emission band at $489 \mathrm{~nm}$. Continuing to add water up to a fraction of $99 \%$, a maximum emission band was shown at $528 \mathrm{~nm}$. The quantum yield of $\mathbf{2}$ was improved as the fraction of water increased from $0 \%$ to 99 and we observed a maximum quantum yield $\left(\Phi_{f}\right)$ of 0.20 at $f_{\mathrm{w}}=99 \%$ (Figure $2, A$ and B). Similarly, the compound $\mathbf{3}$ exhibited a broad emission band range from $470 \mathrm{~nm}$ to $550 \mathrm{~nm}$ with weak emission $\left(\lambda_{\mathrm{em} \max }=\right.$ $504 \mathrm{~nm}$ ) in THF solution, as the water fraction increased from 0 to $80 \%$. The fluorescence was gradually enhanced with two emission peaks at 508 and $542 \mathrm{~nm}$, with a close to 20 -fold enhancement at $f_{\mathrm{w}}$ $=80 \%$ compared with that in pure THF solution (Figure S11), which indicated that both compounds $\mathbf{2}$ and $\mathbf{3}$ are AlE-active materials. The low QY for $\mathbf{3}$ may be caused by $\pi-\pi$ stacking in aggregated state due to the molecule's long $\pi$-conjugated structure and lack of steric protection for the central section of the molecule.

Basically, the Schiff-base containing $\mathrm{CH}=\mathrm{N}$ group exhibits weak fluorescence or non-emissive properties due to the fluorescencequenching photo-induced electron transfer (PET) process. ${ }^{[17]}$ It is noted that the Schiff-base $\mathbf{4}$ emitted a pyrene-like weak, deep blue $\mathrm{FL}\left(\lambda_{\mathrm{em} \max }=426 \mathrm{~nm}\right)$ in pure THF solution upon photoexcitation at $366 \mathrm{~nm}$, and the blue emission probably originated from the strong FL of pyrene units but not the entire molecule scaffold due to the free intramolecular rotation of the substituent aromatic group. As the water fraction increased from 0 to $70 \%$, the emission intensity was dramatically enhanced to its maximum value with a large redshift of $60 \mathrm{~nm}$ to $486 \mathrm{~nm}$, which can be explained by intramolecular charge transfer (ICT). Further increasing the $f_{\mathrm{w}}$ to $99 \%$ leads to emission annihilation (Figure 2, C and D). 
In general, compounds 2-4 show weak emission in pure THF with low quantum yield (less than 0.03), however, as the fraction of water is increased, the emission intensity enhanced at least 30 -fold with an improved quantum yield. What is the dominant physical driving force causing enhancement of emission in the aggregated state? To answer this question, the morphology of 2-4 in THF solution and in $f_{\mathrm{w}}=99 \%$ state were carefully investigated by scanning electron microscopy (SEM) and transmission electron microscopy (TEM). The SEM and TEM of $\mathbf{2}$ are shown in Figure 3. It was found that the compound $\mathbf{2}$ demonstrated a stable gel-like surface in pure THF (Figure $3 \mathrm{~A}$ ) with good crystallinity in a monoclinic system according to the electron diffraction pattern (Figure 3E). As the water fraction increased, the morphology was changed from a gel-like species to small irregular patches and then to uniform, round nanoparticles with sizes of $\sim 60$ and $200 \mathrm{~nm}$ in $f_{\mathrm{w}}$ $=70 \%$ and $99 \%$, respectively (Fig. S19 and Fig. S21), which is in agreement with the DLS measurement. Meanwhile, the crystal system of these corresponding morphologies also changed from monoclinic to amorphous, with enhanced emission and an improved quantum yield. For $\mathbf{4}$, as expected, the morphology also displayed a large difference as the $f_{\mathrm{w}}$ increased (Fig. 4). Specifically, the nanoparticle size in $f_{\mathrm{w}}=70 \%$ of $\mathbf{4}$ was more uniform and larger compared with the others in THF. Furthermore, the nanoparticle size was decreasing slightly, accompanied with a quenching fluorescence and low quantum yield (less than 0.01) when $f_{w}$ was in the range from 70 to $99 \%$. Meanwhile, the high-resolution TEM results showed that the lattice spacing of morphologies 4 in THF solution is about $1.92 \AA$, while in $f_{\mathrm{w}}=99 \%$, the average spacing of lattice fringes became $1.89 \AA$, (Fig. 5). These results correspond to the simulated XRD diffraction peaks at $2 \theta \approx 47^{\circ}$, indicating that 4 in THF at $f_{\mathrm{w}}=99 \%$ remains in the crystalline state. As the water fraction changed from 0 to $99 \%$, the morphology of $\mathbf{4}$ changed from an ordered packing structure (in THF) to amorphous $\left(f_{\mathrm{w}}=70 \%\right)$ and then to the crystalline state $\left(f_{w}=99 \%\right)$. This whole morphology transformation process corresponds with the observed emission enhancement $\left(f_{w}=70 \%\right)$ and fluorescence quenching $\left(f_{w}=99 \%\right)^{[18]}$ On the other hand, the DLS result indicated the nanopaticle size of compound 4 at $f_{\mathrm{w}}=70 \%$ is larger than in pure THF or at $f_{\mathrm{w}}=99 \%$, which corresponds to the highest emission intensity. (Figures S20) Similar SEM and TEM results were also observed for compound $\mathbf{3}$ (Fig. S22-S23).

Therefore, we assumed that the photopyhsical properties in pyrene chemistry vary with the molecular packing pattern, distribution of microstructure, and the nanoparticle size which plays a significant role in enhancing fluorescence intensity. The crystal transition and morphology change from crystalline to amorphous affording a larger uniform regular nanoparticle size, would cause a brighter emission. More importantly, these morphology changes also affect the excited states lifetimes strongly, and both compounds $\mathbf{2}$ and $\mathbf{4}$ show a longer fluorescence lifetime in the nanoparticle compared with the crystalline state.

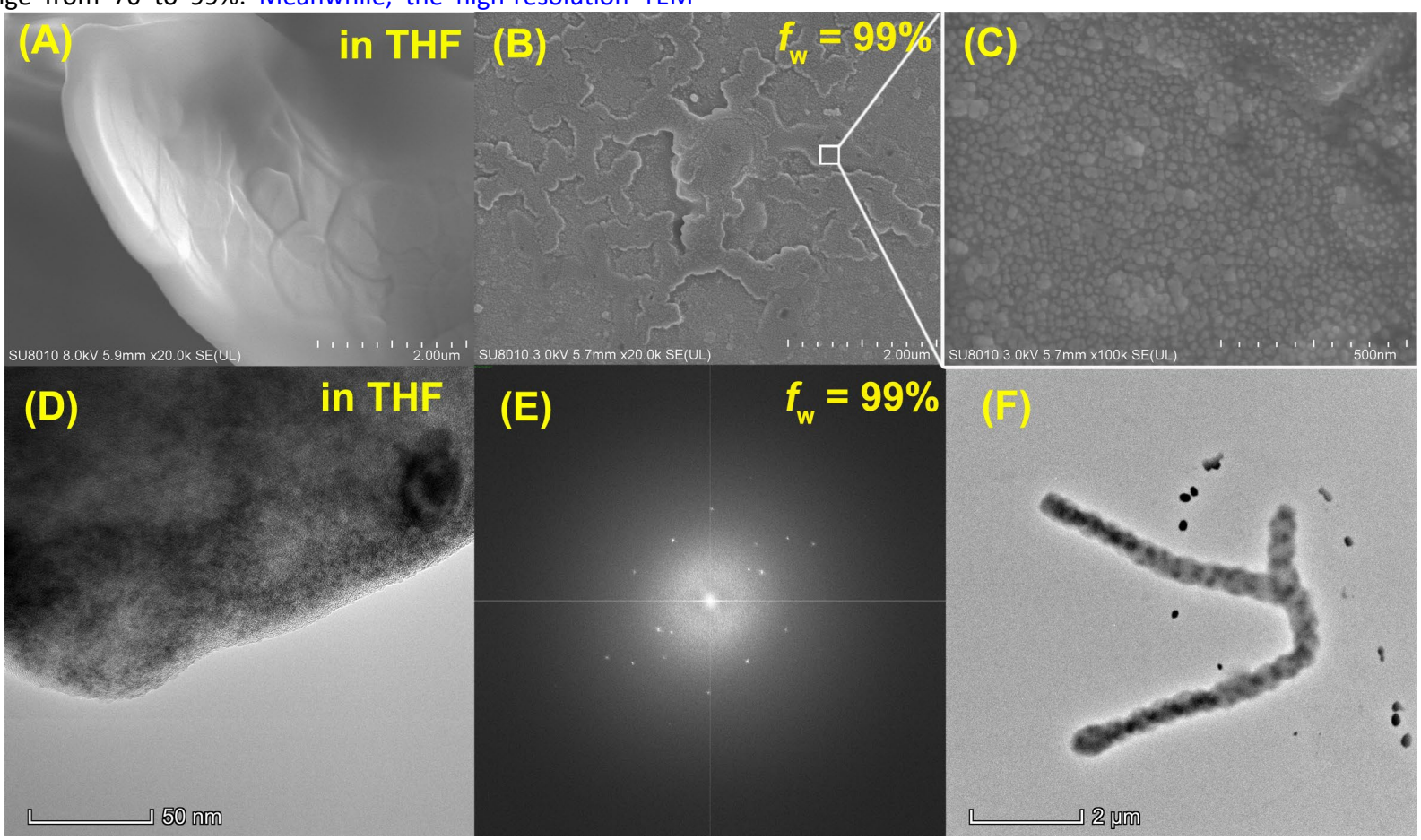

Figure 3. FE-SEM image of self-assembled morphology of $\mathbf{2}(\mathrm{A})$ in THF and (B)in water fraction $f_{\mathrm{w}}=99 \%$, (C) SEM image of the extending regions, (D) TEM image of $\mathbf{2}$ and (E) the corresponding high-resolution TEM images in THF solution, (F) in mixture THF and water with $f_{\mathrm{w}}=$ $99 \%$. 


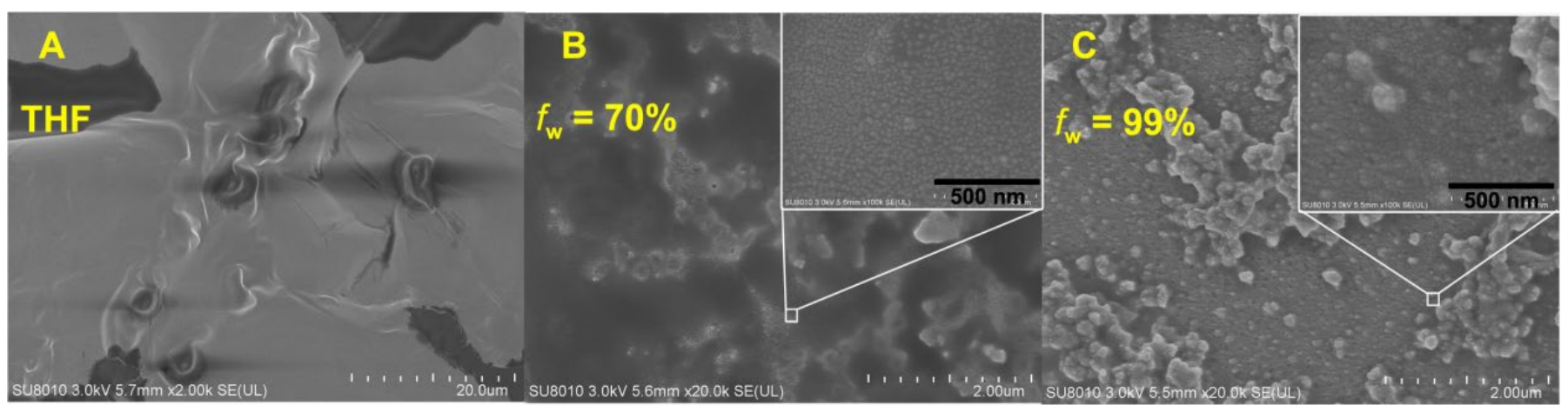

Figure 4. FE-SEM image of self-assembled morphology of 4 (A) in THF; (B) in water fraction $f_{\mathrm{w}}=70 \%$; (C) in water fraction $f_{\mathrm{w}}=99 \%$, insert: SEM image of the extending regions.

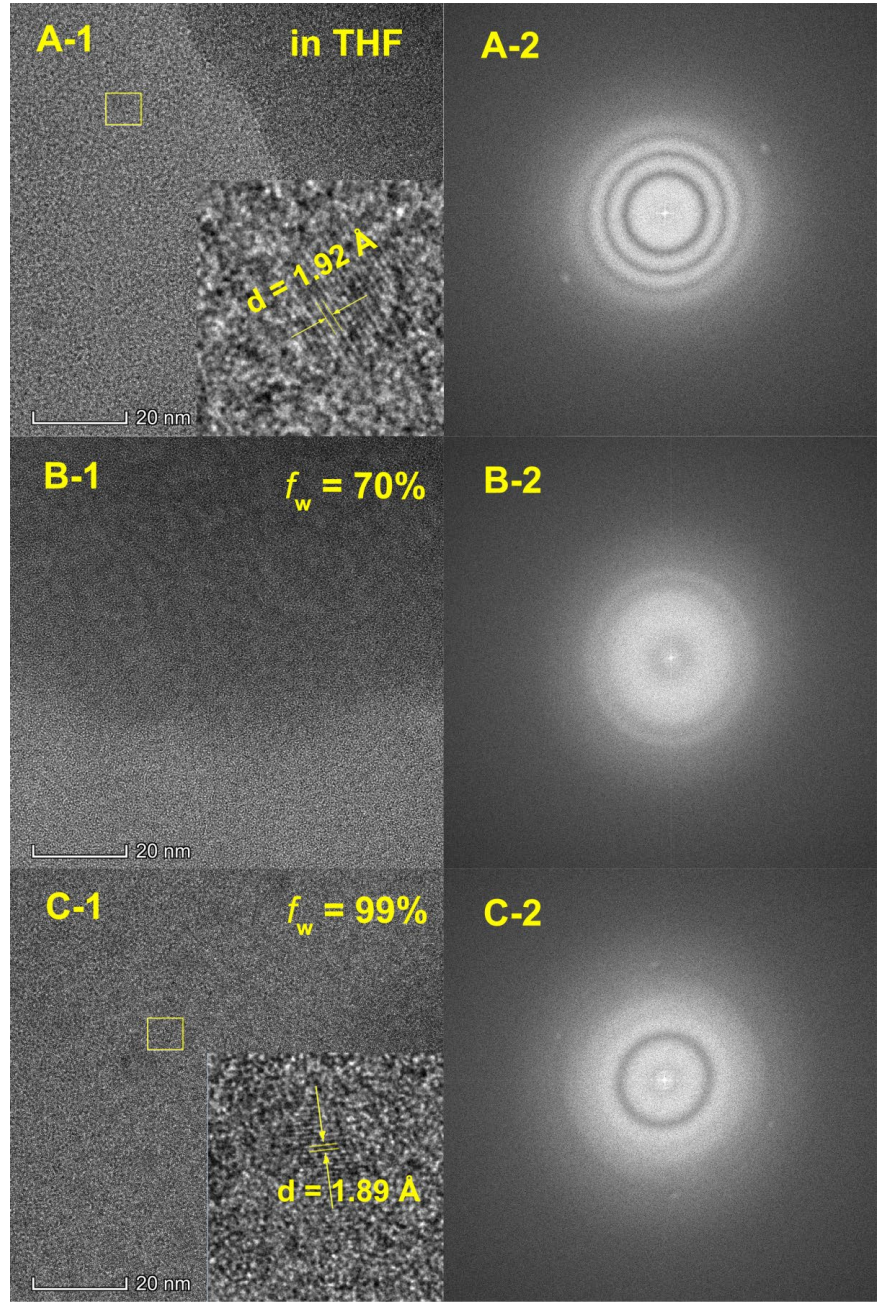

Figure 5. high-resolution TEM images of self-assembled morphology of 4 (A) in THF; (B) in water fraction $f_{\mathrm{w}}=70 \%$; (C) in water fraction $f_{\mathrm{w}}$ $=99 \%$, Insert: HR-TEM image taken from the yellow square and the corresponding (A-2, B-2 and C-2) selected-area electron diffraction (SAED) pattern.

\section{X-ray single crystal diffraction analysis}

The conformations of pyrene derivatives 2 and $\mathbf{4}$ in the crystalline state were determined by X-ray single crystal diffraction. Both suitable crystals for X-ray measurements were grown in THF-hexane binary solvent mixtures. Despite many attempts we were unable to grow single crystals of $\mathbf{3}$.
The crystallographic information is summarized in table 2 . The $\mathrm{X}$-ray single crystal structure of $\mathbf{2}$ is monoclinic with space group $P 2_{1} / c$. As shown in Figure 6 , the pyrene derivatives containing phenylacetonitrile moieties possess a highly twisted conformation between the pyrene ring and the phenyl group with a dihedral angle of $58.50(6)^{\circ}$. The bulky tert-butyl groups play an important role in suppressing $\pi-\pi$ stacking in the solid state of pyrene derivatives. However, according to the crystal packing in 2, two different packing patterns were observed, namely Patten A (b) and Patten B (c). It is obvious that the pyrene core adopts face-to-face $\pi-\pi$ stacking to form a dimer with a separation of $c a$. $3.74 \AA$ in pattern $A$, and the dimer motif was further strengthened by strong intermolecular interactions via several $\mathrm{C}-\mathrm{H} \cdots \pi$ bonds $\left(\mathrm{C} 20-\mathrm{H} 20 \mathrm{~A} \cdots \mathrm{C}^{\prime} 2^{\prime}=2.88\right.$ $\AA$ and $\mathrm{C} 18-\mathrm{H} 18 \mathrm{C} \cdots \mathrm{C} 10^{\prime}=2.97 \AA$ ) (Figures $6 \mathrm{~b}$ and $6 \mathrm{c}$ ). On the other hand, in pattern $B$, two neighbouring pyrene moieties are present with an edge-to-edge motif with $\mathrm{C}-\mathrm{H} \cdots \pi$ noncovalent interactions $\left(\mathrm{C} 25-\mathrm{H} 25 \cdots \mathrm{C}^{\prime}{ }^{\prime}=3.14 \AA\right.$ A) (Figure 4d), giving a one dimensional (1D) packing architecture involving alternating patterns $A$ and $B$ along the $c$ axis (Figure 6e).
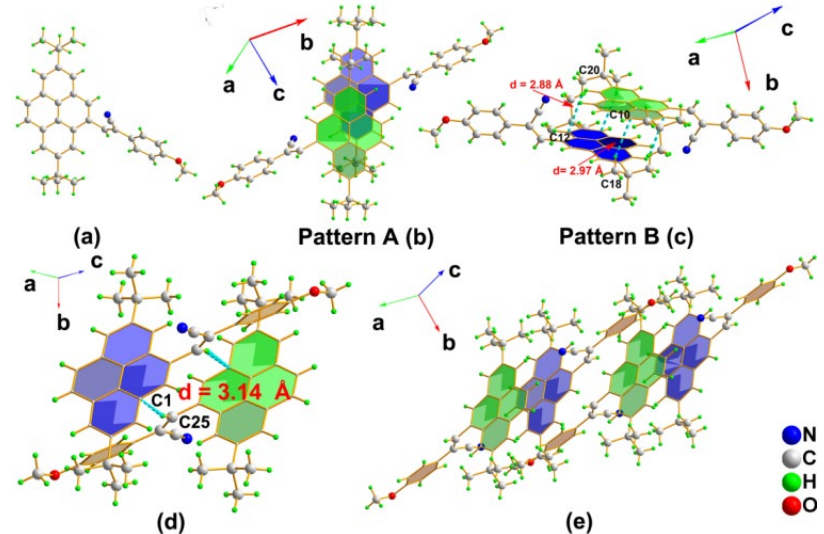

Figure 6. (a) X-ray single crystal structure of 2 , and face-to-face $\pi-\pi$ stacking of pyrene moieties in packing structure (b) top view, (c) side view, (d) multiple weak $\mathrm{C}-\mathrm{H}$... $\pi$ interactions between adjacent molecules with distance $c a$. $3.14 \AA$, (e) 1D supramolecular chains. 


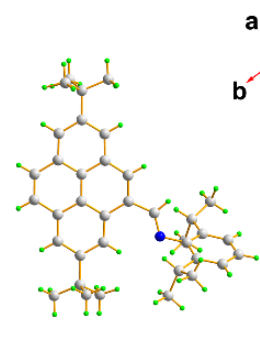

(a)

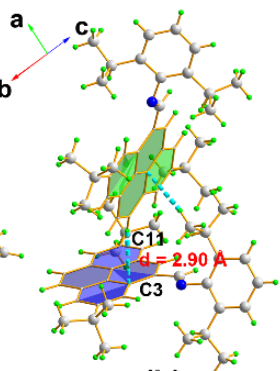

(b)

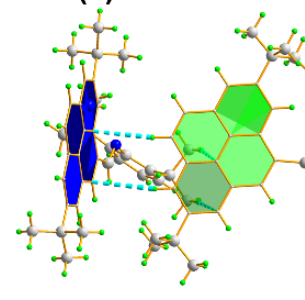

(d)

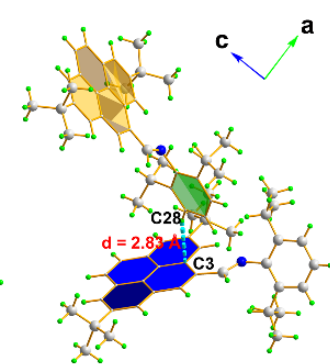

(c)

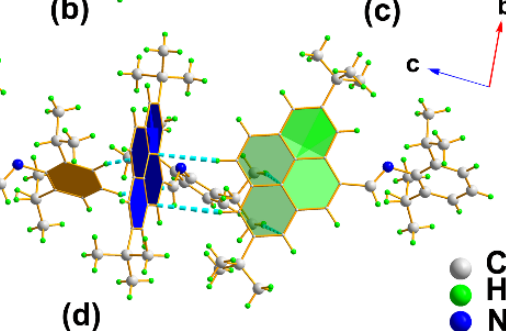

Figure 7. (a) X-ray single crystal structure of 4, (b) and (c) two types of packing arrangements and (d) a 1D supramolecular chain connected by weak $\mathrm{C}-\mathrm{H} \cdots \pi$ interactions.

The Schiff-base $\mathbf{4}$ crystallizes in the orthorhombic centrosymmetric space group Pccn and is shown in Figure 7. A large twist angle was found between the central pyrene fragment and the phenyl ring with a dihedral angle of $62.9(7)^{\circ}$ due to the steric effect of the isopropyl groups on the substituent at the 4-position of pyrene. Both the ${ }^{t} \mathrm{Bu}$ groups of pyrene at $\mathrm{C}(17)$ and the ${ }^{i} \mathrm{Pr}$ group at $\mathrm{C}(35)$ show 2 -fold disorder with occupancy ratio 0.615:0.385(6) for $\mathrm{C} 18, \mathrm{C} 19$, and $\mathrm{C} 20$, and 0.578:0.422(16) for C36 and C37. Indeed, the most dominant intermolecular interactions in the Schiff-base 4 crystal packing are the edge-to-face $\mathrm{C}-\mathrm{H} \cdots \pi$ interactions $\left(\mathrm{C} 36-\mathrm{H} 36 \mathrm{~B} \cdots \mathrm{C} 1^{\prime}=\right.$ $2.83 \AA, \mathrm{C} 28-\mathrm{H} 28 \cdots \mathrm{C}^{\prime}=2.90 \AA, \mathrm{C} 11-\mathrm{H} 11 \cdots \mathrm{C}^{\prime}=2.90 \AA$, and $\mathrm{C} 29-\mathrm{H} 29 \cdots \mathrm{C} 25=2.78 \AA$ ) giving a herringbone motif, but no $\pi-\pi$ stacking exists in either packing pattern A or B. As shown in Figure $7 d$, a basic 1D supramolecular chain was constructed though weak $\mathrm{C} 36-\mathrm{H} 36 \cdots \mathrm{C}^{\prime}$ interactions along the $c$ axis.

Table 2. Summary of crystal data for pyrene-based AlEgens $\mathbf{2}$ and $\mathbf{4}$.

\begin{tabular}{|c|c|c|}
\hline Parameter & 2 & 4 \\
\hline Empirical formula & $\mathrm{C}_{34} \mathrm{H}_{33} \mathrm{NO}$ & $\mathrm{C}_{37} \mathrm{H}_{43} \mathrm{~N}$ \\
\hline $\begin{array}{l}\text { Formula weight } \\
\qquad\left[\mathrm{g} \mathrm{mol}^{-1}\right]\end{array}$ & 471.61 & 501.72 \\
\hline Crystal system & monoclinic & orthorhombic \\
\hline Space group & $P 2_{1} / c$ & Pccn \\
\hline$a[\AA ̊]$ & $10.6101(9)$ & 12.9754 (19) \\
\hline$b[\AA]$ & $22.3235(15)$ & $36.630(5)$ \\
\hline$c[\AA]$ & $11.4058(8)$ & $12.4364(18)$ \\
\hline$B\left[^{\circ}\right]$ & $98.578(2)$ & 90 \\
\hline Volume $\left[\AA^{3}\right]$ & $2671.3(3)$ & $5910.9(15)$ \\
\hline$Z$ & 4 & 8 \\
\hline $\begin{array}{l}\text { Density, calcd } \\
{\left[\mathrm{Mg} \mathrm{m}^{-3}\right]}\end{array}$ & 1.173 & 1.128 \\
\hline Temperature $[\mathrm{K}]$ & 203 & 150 \\
\hline Unique reflns & 4795 & 5399 \\
\hline Obsd reflns $[I>2 \sigma(I)]$ & 2407 & 3352 \\
\hline Parameters & 358 & 395 \\
\hline
\end{tabular}

\begin{tabular}{ccc}
\hline$R_{\text {int }}$ & 0.062 & 0.061 \\
$R[\mid>2 \sigma(I)]^{\mathrm{a}}$ & 0.053 & 0.062 \\
$w R[\mid>2 \sigma(I)]^{\mathrm{b}}$ & 0.156 & 0.187 \\
$\mathrm{GOF}$ on $F^{2}$ & 1.01 & 1.04 \\
\hline${ }^{2}$ Conventional $R$ on $F_{\mathrm{hkl}}: \Sigma|| F_{\mathrm{o}}|-| F_{\mathrm{c}}|| / \sigma\left|F_{\mathrm{o}}\right| .{ }^{\mathrm{b}}$ Weighted $R$ &
\end{tabular}

\section{Mechanochromism Experiments}

Pyrene and its derivatives are being considered as ACQ compounds but few reports have been found to show MC behaviour. ${ }^{[5 b, 19]}$ To test their possible MC properties, pyrene luminogens 2-4 as a solid (crystals) were ground in an agate mortar. The maximum emission peak of $\mathbf{2}$ gave a hypsochromic shift from $508 \mathrm{~nm}$ to $502 \mathrm{~nm}$ after slight grinding. But after heavy grinding, the emission peak showed a larger red-shift to $512 \mathrm{~nm}$ (Figure 8A), while the emission of $\mathbf{3}$ did not show an emission band shift after grinding (Figure S17). The results indicated that compound $\mathbf{2}$ is $\mathrm{MC}$-active and compound $\mathbf{3}$ is inactive. In addition, the fluorescence quantum yield $\left(\Phi_{f}\right)$ of both samples $\mathbf{2}$ and $\mathbf{3}$ was higher after grinding (see Table 1). The microstructure of the pyrene luminogens was further investigated by powder X-ray diffraction (PXRD). As Figure S24 shows, the XRD pattern of crystal $\mathbf{2}$ is almost the same as the simulated XRD powder pattern. After grinding, the intensity of the diffraction peaks was decreased with a wider peak width at half height (FWHM), indicating that the ground compound 2 still shows microcrystalline order with a reduced crystal size according to the Scherrer equation $(D=k \lambda / \cos \theta)$. ${ }^{[20]}$ The main differences between them are that the corresponding $d$ spacing decreased $(d=11.157 \AA$ for crystal 2 and $d=11.113 \AA$ $\mathrm{nm}$ for the ground compound), which was defined by the Miller plane ( $\mathrm{k} \mathrm{kl=0} 20$ ), ${ }^{[21]}$ from the diffraction peaks at $2 \theta=$ 7.42 and $7.81^{\circ}$, meaning that the twisted molecules tend to be more planer and the molecular arrangement becomes closer with stronger intermolecular interactions, which would cause the enhanced emission. Furthermore, the calculated crystal system of ground compound $\mathbf{2}$ remains monoclinic. On the other hand, the PXRD studies (Figure S25) on compound 3 showed similar weak XRD patterns before and after the grinding, which suggested that both states displayed a similar molecular packing structure, and consequently displayed $\mathrm{PL}$ behaviour with almost same QY $(0.1 \& 0.14)$. We inferred that this is due to the substituent and pyrene core in compound $\mathbf{3}$ having a more co-planar structure and the molecular configuration undergoes a limited change under external force. Although we failed to grow suitable crystals for $\mathbf{3}$, the optimized molecular geometry of 2-4 in the gas phase was performed at the B3LYP/6-31G* level using Gaussian 03 software. Indeed, the dihedral angle of $\mathbf{3}$ between phenylacetonitrile moieties and the pyrene core is $17.6^{\circ}$, which is less than in $\mathbf{2}$. The small dihedral angle probably leads to face-to-face interactions with neighbouring pyrenes in the aggregated state. 
Table 3 Summarized XRD parameters for compound $\mathbf{2}$

\begin{tabular}{ccccc}
\hline Compound & simulated $^{\mathrm{a}}$ & crystal $^{\mathrm{a}}$ & Grind $^{\mathrm{a}}$ & Heavy grind $^{\mathrm{a}}$ \\
\hline Crystal system & monoclinic & monoclinic & monoclinic & monoclinic \\
$a[\AA \AA]$ & $10.6146(2)$ & $10.64(1)$ & $10.656(5)$ & $10.639(8)$ \\
$b[\AA \AA]$ & $22.3317(9)$ & $22.31(2)$ & $22.355(8)$ & $22.22(1)$ \\
$c[\AA]$ & $11.4084(2)$ & $11.50(1)$ & $11.398(5)$ & $11.451(8)$ \\
$B\left[^{\circ}\right]$ & $98.535(2)$ & $98.79(7)$ & $98.31(4)$ & $98.64(6)$ \\
Crystallinity (\%) & 99.80 & 99.88 & 98.97 & 99.81 \\
$\mathbf{2} \theta\left(^{\circ}\right)$ & 7.906 & 7.773 & 7.805 & 7.806 \\
FWHM (०) & 0.173 & 0.152 & 0.419 & 0.371 \\
a fitted based on crystal & & & \\
\hline
\end{tabular}
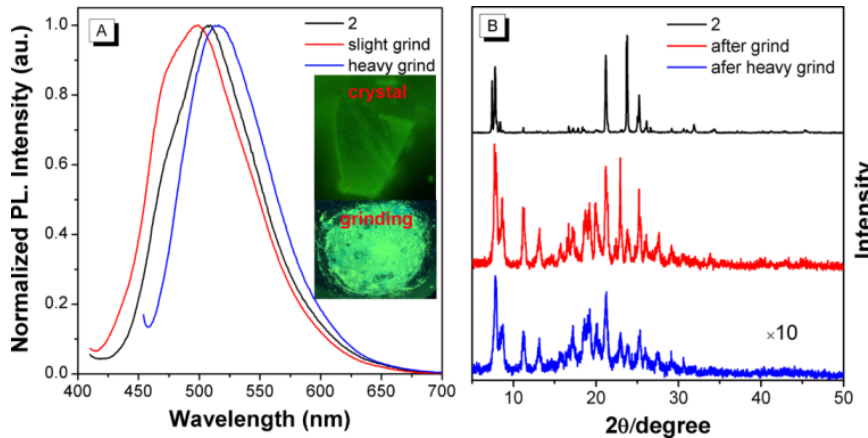

Figure 8 (A) The PL spectrum of $\mathbf{2}$ before and after grinding, insert: The images of $\mathbf{2}$ in the crystalline state and after grinding taken under $365 \mathrm{~nm}$ UV light. (B) Wide angle XRD diffractograms of $\mathbf{2}$ in different states.

It is noted that the compound $\mathbf{4}$ shows no emission in the solid state. The crystal and powder samples of $\mathbf{4}$ were characterized by X-ray diffraction analysis and PXRD (Figure S26). In the single crystal structure of 4 , the large twist angle between the pyrene core and the isopropyl-bearing aromatic plane of the substituent group is $62.9(7)^{\circ}$, According to the XRD patterns, we calculated the corresponding $d$-spacings are $18.896 \AA$ for crystal 4 and $d=18.455 \AA$ for the powder, from the diffraction peaks at $2 \vartheta=4.67^{\circ}$ and $4.78^{\circ}$ (h $\mathrm{kl}=020$ ), which indicated the well-ordered crystal structure becomes more planar after grinding, but the crystal system still remains orthorhombic with a larger FWHM.

\section{Experimental}

Characterization. ${ }^{1} \mathrm{H}$ and ${ }^{13} \mathrm{C}$ NMR spectra $(400 \mathrm{MHz})$ were recorded on a Bruker AV 400 spectrometer using chloroform- $d$ solvent and tetramethylsilane as internal reference. $J$-values are given in $\mathrm{Hz}$. High-resolution mass spectra (HRMS) were taken on a GCT premier CAB048 mass spectrometer operating in a MALDI-TOF mode. UV-vis absorption spectra and Photoluminescence (PL) spectra were recorded on a Shimadzu UV-2600 and a Perkin-Elmer LS 55 fluorescence spectrometer. PL quantum yields were measured using absolute methods using a Hamamatsu C11347-11 Quantaurus-QY Analyzer. The lifetime was recorded on an Edinburgh FLS 980 instrument and measured using a time correlated single-photon counting method. Scanning electron microscopy (SEM) images were obtained using a Hitachi scanning electron microscope. The transmission electron microscopy (TEM) images were obtained on a transmission electron microscope (Thermo, Talos F200S) at an accelerating voltage of $200 \mathrm{kV}$. Powder XRD patterns were recorded on a Rigaku D/MAX-Ultima IV X-ray diffractometer, using $\mathrm{Cu} K \alpha$ radiation $(\lambda=1.54 \AA$, $40 \mathrm{~mA}, 40 \mathrm{mV})$. Cyclic voltammetry was carried out in $0.10 \mathrm{M}$ tetrabutylammonium perchlorate in anhydrous dichloromethane and THF at a scan rate of $100 \mathrm{mVs}^{-1}$ at room temperature. Thermogravimetric analysis was carried on a Mettler ToledoTGA/DSC3+ under dry nitrogen at a heating rate of $10^{\circ} \mathrm{C} / \mathrm{min}$. The quantum chemistry calculation was performed on the Gaussian 09W (B3LYP/6-31G* basis set) software package. ${ }^{[22]}$

X-ray Crystallography. Crystallographic data for $\mathbf{2}$ and $\mathbf{4}$ were collected on a Bruker APEX 2 CCD diffractometer with graphitemonochromated Mo K $\alpha$ radiation $(\lambda=0.71073 \AA)$ in the $\omega$ scan mode. ${ }^{[23,24]}$ The structures were solved by a charge flipping algorithm and refined by full-matrix least-squares methods on $F^{2}$. [25] All esds were estimated using the full covariance matrix. Further details are presented in Table 2 . In $\mathbf{2}$ the methyl groups on the ${ }^{t} \mathrm{Bu}$ group at $C(21)$ were modelled as disordered over two sets of positions with major occupancy factor $81.0(6) \%$. In 4 the ${ }^{t} \mathrm{Bu}$ group at $C(17)$ was similarly modelled with major component $61.5(6) \%$ and the $\operatorname{Pr}$ group at $\mathrm{C}(35)$ had its Me groups modelled as two-fold disordered with major component 57.8(16)\%. Data for the structures reported here has been deposited with the Cambridge Crystallographic Data Centre with deposition numbers. CCDC 1886231-2 contain the supplementary crystallographic data for this paper. These data can be obtained free of charge from The Cambridge Crystallographic Data Centre via www.ccdc.cam.ac.uk/data request/cif.

Materials: Unless otherwise stated, all reagents were purchased from commercial sources and used without further purification. Tetrahydrofuran was distilled prior to use. 2,7-di-tert-butylpyrene4-carbaldehyde (1) was prepared as described previously. ${ }^{[26]}{ }^{1} \mathrm{H}$ NMR (400 MHz, $\left.\mathrm{CDCl}_{3}\right) \delta 10.54(\mathrm{~s}, 1 \mathrm{H}), 9.73(\mathrm{~d}, J=1.6 \mathrm{~Hz}, 1 \mathrm{H}), 8.59$ 
(s, 1H), 8.36 (d, $J=5.1 \mathrm{~Hz}, 2 \mathrm{H}), 8.28$ (d, $J=1.5 \mathrm{~Hz}, 1 \mathrm{H}), 8.05$ (q, $J=$ $8.9 \mathrm{~Hz}, 2 \mathrm{H}), 1.61(\mathrm{~s}, 9 \mathrm{H}), 1.60(\mathrm{~s}, 9 \mathrm{H})$.

Synthesis of (Z)-3-(2,7-di-tert-butylpyren-4-yl)-2-(4methoxyphenyl) acrylonitrile (2) A mixture of 2,7-di-tertbutylpyrene-4-carbaldehyde (342 mg, $1 \mathrm{mmoL}, 1.0 \mathrm{eq}$ ), (4methoxyphenyl) acetonitrile (176 mg, $1.2 \mathrm{mmoL}, 1.2 \mathrm{eq}$ ) and potassium tert-butoxide $(336 \mathrm{mg}, 3 \mathrm{mmoL}, 3 \mathrm{eq})$ in ethanol $(12 \mathrm{~mL})$ was stirred and refluxed overnight under nitrogen. After cooling to room temperature, the mixture was filtered and washed with ethanol three times. The residue was further crystallized from THF and hexane to give yellow block crystals (302 mg, yield $64 \%$ ). ${ }^{1} \mathrm{H}$ NMR $\left(400 \mathrm{MHz}, \mathrm{CDCl}_{3}\right) \delta 8.62(\mathrm{~s}, 1 \mathrm{H}), 8.33(\mathrm{~s}, 2 \mathrm{H}), 8.30-8.22(\mathrm{~m}$, $3 \mathrm{H}), 8.07(\mathrm{~d}, J=0.5 \mathrm{~Hz}, 2 \mathrm{H}), 7.96-7.76(\mathrm{~m}, 2 \mathrm{H}), 7.07(\mathrm{~d}, J=8.8 \mathrm{~Hz}$, 2H), $3.90(\mathrm{~s}, 3 \mathrm{H}), 1.61$ (dd, $J=6.6,2.0 \mathrm{~Hz}, 18 \mathrm{H}) .{ }^{13} \mathrm{C} \mathrm{NMR}(100 \mathrm{MHz}$, $\left.\mathrm{CDCl}_{3}\right) \delta 160.05,148.57,148.12,138.09,130.61,130.40,130.05$, $129.16,128.60,128.55,127.12,127.04,126.89,126.33,123.00$, $122.87,122.48,122.35,122.28,117.49,117.45,114.31,113.99$, $54.89,34.82,34.68,31.41,31.33$. HRMS (MALDI-TOF): $\mathrm{m} /$ zcalcd for $\mathrm{C}_{34} \mathrm{H}_{33} \mathrm{NO} 471.26$; found $471.2607\left[\mathrm{M}^{+}\right]$.

Synthesis of (2Z,2'Z)-2,2'-(1,4-phenylene) bis(3-(2,7-di-tertbutylpyren-4-yl) acrylonitrile) (3) A mixture of 2,7-di-tertbutylpyrene-4-carbaldehyde (548 mg, $1.6 \mathrm{mmoL}, 2.5 \mathrm{eq}$ ), 1,4benzenediacetonitrile $(100 \mathrm{mg}, 0.64 \mathrm{mmoL}, 1.0 \mathrm{eq})$ and potassium tert-butoxide (431 $\mathrm{mg}, 3.84 \mathrm{mmoL}, 3 \mathrm{eq}$ ) in ethanol $(15 \mathrm{~mL}$ ) was stirred and refluxed overnight under nitrogen. After cooling to room temperature, the mixture was filtered and washed with ethanol three times. The residue was further crystallized from $\mathrm{CHCl}_{3}$ and hexane to give an orange powder (224 mg, yield $43 \%$ ). HRMS (MALDI-TOF): $\mathrm{m} /$ zcalcd for $\mathrm{C}_{60} \mathrm{H}_{56} \mathrm{~N}_{2}$ 804.44; found 804.4345 [M+'].

\section{Synthesis of (E)-1-(2,7-di-tert-butylpyren-4-yl)-N-(2,6-} diisopropylphenyl)methanimine (4) A mixture of 2,7-di-tertbutylpyrene-4-carbaldehyde (342 mg, $1 \mathrm{mmoL}, 1.0 \mathrm{eq}$ ), 2,6diisopropylaniline ( $212 \mathrm{mg}, 1.2 \mathrm{mmoL}, 1.2 \mathrm{eq}$ ) and potassium tertbutoxide $(340 \mathrm{mg}, 3 \mathrm{mmoL}, 3 \mathrm{eq})$ in ethanol $(10 \mathrm{~mL}$ ) was stirred overnight at room temperature under nitrogen. The mixture was filtered and washed with methanol. The residue was further crystallized from $\mathrm{CH}_{2} \mathrm{Cl}_{2}$ and hexane to give yellow needle crystals (254 mg, yield $51 \%$ ). ${ }^{1} \mathrm{H}$ NMR $\left(400 \mathrm{MHz}, \mathrm{CDCl}_{3}\right) \delta 9.77(\mathrm{~d}, J=1.3 \mathrm{~Hz}$, $1 \mathrm{H}), 9.01(\mathrm{~s}, 1 \mathrm{H}), 8.56(\mathrm{~s}, 1 \mathrm{H}), 8.33(\mathrm{dd}, J=19.1,1.6 \mathrm{~Hz}, 3 \mathrm{H}$ ), 8.09 (q, $J=8.9 \mathrm{~Hz}, 2 \mathrm{H}), 7.30(\mathrm{~d}, J=7.2 \mathrm{~Hz}, 2 \mathrm{H}), 7.22(\mathrm{dd}, J=8.5,6.7 \mathrm{~Hz}, 1 \mathrm{H})$, $3.39-3.19(\mathrm{~m}, 2 \mathrm{H}), 1.66-1.54(\mathrm{~m}, 18 \mathrm{H}), 1.30(\mathrm{~d}, J=6.9 \mathrm{~Hz}, 12 \mathrm{H})$. ${ }^{13} \mathrm{C} \mathrm{NMR}\left(100 \mathrm{MHz}, \mathrm{CDCl}_{3}\right) \delta 162.99,149.52,148.78,148.41,137.17$, $133.45,130.40,130.18,130.12,129.10,127.91,127.53,126.41$, $123.52,123.43,123.00,122.89,122.56,122.08,120.17,34.96$, $34.64,31.38,31.29,27.57,22.99$. HRMS (MALDI-TOF): $\mathrm{m} / \mathrm{zcalcd}$ for $\mathrm{C}_{37} \mathrm{H}_{43} \mathrm{~N}$ 501.34; found $501.3533\left[\mathrm{M}^{+}\right]$.

\section{Conclusions}

In summary, three pyrene-based AlEgens 2-4 were designed and synthesized to investigate the relationship between the morphology and fluorescence properties as well as mechanochromism (MC) properties. A large, planar pyrene core is present in non-symmetric luminogen $\mathbf{2}$, which displayed clear MC properties by introducing a twisted cyanoethylene moiety however, homologue $\mathbf{3}$ was MC-inactive due to the extended $\pi$-conjugation with an almost planar scaffold. Whereas, the Schiff-base $\mathbf{4}$ shows weak/non-emissive properties in pure THF or in the solid state due to PET effect. But the fluorescence intensity increased as the water fraction $\left(f_{w}\right)$ increased to $70 \%$. The FE-SEM results indicated the morphological transformation of pyrene-based AIEgens from irregular shaped to uniform nanoparticle species with larger size, and the crystal phase changed to amorphous, which enhanced emission. Furthermore, the single crystal X-ray diffraction and PXRD of $\mathbf{2}$ and $\mathbf{4}$, proved that both $\mathbf{2}$ and $\mathbf{4}$ have twisted molecular geometries but become more planar under external pressure which caused a clear MC phenomenon with a tuneable emission band. The article presents, for the first time, a correlation between molecular structure, particle size distribution and fluorescence properties in pyrene systems, and this research would open a new door for developing novel, high-performance pyrene-based luminogens with $M C$ properties for potential applications.

\section{Conflicts of interest}

There are no conflicts to declare.

\section{Acknowledgements}

This work was supported by and the National Natural Science Foundation of China (21602014, 51620105009, 11674310 and 21788102), the Youth Fund of Guangdong University of Technology (1108-220413205) and Guangdong provincial key laboratory of functional soft condensed matter. M. M. Islam thanks to MOST, P. R. China for Talented Young Scientist Program (TYSP) Fellowship. We also thank the technical support from AIEgen Biotech Co., Ltd.

\section{Notes and references}

1 (a) T. M. Figueira-duarte and M. Klaus, Chem. Rev., 2011 , 111, 7260; (b) A. Mateo-Alonso, Chem. Soc. Rev., 2014, 43, 6311.

2 (a) X. Feng, J. Y. Hu, C. Redshaw and T Yamato, Chem. Eur. J., 2016, 22, 11898; (b) X. Feng, Y. Du, X.-F. Wei and T. Yamato, China Science and Technology Press, 2016.

3 J. Luo, Z. Xie, J. W. Y. Lam, L. Cheng, H. Chen, C. Qiu, H. S. Kwok, X. Zhan, Y. Liu, D. Zhu and B. Z. Tang, Chem. Comm., 2001, 1740-1741.

4 Y. Hong, J. W. Y. Lam and B. Z. Tang, Chem. Soc. Rev., 2011, 40, 5361-5388.

5 (a) B. Pramanik and D. Das, J. Phys. Chem. C, 2018, 122, 3655 (b) M. M. Islam, Z. Hu, Q. Wang, C. Redshaw and X. Feng, Mater. Chem. Front., 2019, DOI: 10.1039/C9QM00090A

6 Z. Zhao, S. Chen, J. W. Y. Lam, P. Lu, Y. Zhong, K. S. Wong, H. S. Kwok and B. Z. Tang, Chem. Commun., 2010, 46, 2221. 
7 Z. Zhao, S. Chen, J. W. Y. Lam, Z. Wang, P. Lu, F. Mahtab, H. H. Y. Sung, I. D. Williams, Y. Ma, H. S. Kwok and B. Z. Tang, J. Mater. Chem., 2011, 21, 7210.

8 (a) R. Tan, S. Wang, H. Lan, S. Xiao, Curr. Org. Chem., 2017, 21, 236; (b) Y. Xie and Z. Li, Chem, 2018, 4, 943.

9 Y.-B. Gong, P. Zhang, Y.-R. Gu, J.-Q. Wang, M.-M. Han, C. Chen, X.-J. Zhan, Z.-L. Xie, B. Zou, Q. Peng, Z.-G. Chi and Z. Li, Adv. Optical Mater., 2018, 1800198.

10 J. Katla, H. R. Bhat, P. C. Jha, P. S. Ghalsasi and S. Kanvah, ChemistrySelect, 2017, 2, 1902.

11 (a) J. Zhao, Z. Chi, Z. Yang, Z. Mao, Y. Zhang, E. Ubba and Z. Chi, Mater. Chem. Front., 2018, 2, 1595; (b) K. Kokado and K. Sada, 2019, DOI: : 10.1002/anie.201814462.

12 X. Feng, J. Y. Hu, L. Yi, N. Seto, Z. Tao, C. Redshaw, M. R. J. Elsegood and T. Yamato, Chem. Asian J., 2012, 7, 2854.

13 D. Dang, Z. Qiu, T. Han, Y. Liu, M. Chen, R. T. K. Kwok, J. W. Y. Lam and B. Z. Tang, Adv. Funct. Mater., 2018, $28,1$.

14 (a) J. Yang, Q. Guo, X. Wen, X. Gao, Q. Peng, Q. Li, D. Ma, Z. Li, J. Mater. Chem. C, 2016, 4, 8506; (b) J. Yang, L. Li, Y. Yu, Z. Ren, Q. Peng, S. Ye, Q. Li and Z. Li, Mater. Chem. Front., 2017, $1,91$.

15 (a) U. Diwan, V. Kumar, R. K. Mishra, N. K. Rana, B. Koch, M. K. Singh and K. K. Upadhyay, Anal. Chim. Acta, 2016, 929, 39; (b) H. Xiao, K. Chen, D. Cui, N. Jiang, G. Yin, J Wang and R. Wang, New J. Chem., 2014, 38, 2386; (c) J. Cheng, K. Wei, X. Ma, X. Zhou and H. Xiang, J. Phys. Chem. C, 2013, 117, 16552.

16 (a) C. Z. Wang, Y. Noda, C. Wu, X. Feng, P. Venkatesan, H Cong, M. R. J. Elsegood, T. G. Warwick, S. J. Teat, C. Redshaw and T. Yamato, Asian J. Org. Chem., 2018, 7, 444; (b) M. Shyamal, P. Mazumdar, S. Maity, G. P. Sahoo, G. SalgadoMorán and A. Misra, J. Phys. Chem. A, 2016, 120, 210.

17 (a) A. Kathiravan, K. Sundaravel, M Jaccob, G. Dhinagaran, A. Rameshkumar, D. A. Ananth and T. Sivasudha, J. Phys. Chem $B, 2014,118,13573$; (b) S. T. Manjare, Y. Kim and D. G. Churchill, Acc. Chem. Res., 2014, 47, 2985.

18 X. He, Z. Zhao, L.-H. Xiong, P. F. Gao, C. Peng, R. S. Li, Y. Xiong, Z. Li, H. H.-Y. Sung, I. D. Williams, R. T. K. Kwok, J. W. Y. Lam, C. Zhi Huang, N. Ma and B. Z. Tang, J. Am. Chem. Soc., 2018, 140, 6904.

19 (a) W. Li, L. Wang, J. P. Zhang and H. Wang, J. Mater. Chem. C 2014, 2, 1887; (b) W. Li, P. P. Yang, L. Wang, H. Wang, J. Mater. Chem. C, 2015, 3, 3783.

20 (a) JIS R 7651. Measurement of lattice parameters and crystallite sizes of carbon materials; 2007; (b) N. Iwashita, M Inagaki, Relations between structural parameters obtained by X-ray powder diffraction of various carbon materials, Carbon, 1993, 31, 1107; (c) W. Gao, Y. Li, J. Guo, M. Ni, M. Liao, H. Mo and J. Li, Nanoscale, 2018, 10, 8787.

21 (a) Bruker TOPAS, 7; Bruker AXS Inc.: Karlsruhe, Germany, 2017; (b) Cheary, R. W.; Coelho, A. A., A fundamental parameters approach to X-ray line-profile fitting. J. Appl. Cryst. 1992, 25, 109.

22 M. J. Frisch, Gaussian 09, revision A. 02; Gaussian, Inc., Pittsburgh, PA, 2009,

23 SAINT and SADABS; Bruker AXS Inc.: Madison, Wisconsin, USA, 2005.

24 SHELXTL Program Package, version 5.1; Bruker AXS, Inc: Madison, WI, 1997.

25 Sheldrick, G. M. Acta Crystallogr. 2008, A64, 112.

26 J. Y. Hu, A. Paudel, N. Seto, X. Feng, M. Era, T. Matsumoto, J. Tanaka, M. R. J. Elsegood, C. Redshaw and T. Yamato, Org. Biomol. Chem., 2013, 11, 2186. 